\title{
Anxiety and cognitive efficiency: Differential modulation of transient and sustained neural activity during a working memory task
}

\author{
C. L. FAles AND D. M. Barch \\ Washington University, St. Louis, Missouri \\ G. C. Burgess \\ University of Colorado, Boulder, Colorado \\ A. SCHAEFER \\ University of Leeds, Leeds, England \\ D. S. Mennin and J. R. Gray \\ Yale University, New Haven, Connecticut \\ AND \\ T. S. BRAVER \\ Washington University, St. Louis, Missouri
}

\begin{abstract}
According to the processing-efficiency hypothesis (Eysenck, Derakshan, Santos, \& Calvo, 2007), anxious individuals are thought to require greater activation of brain systems supporting cognitive control (e.g., dorsolateral prefrontal cortex; DLPFC) in order to maintain equivalent performance to nonanxious subjects. A recent theory of cognitive control (Braver, Gray, \& Burgess, 2007) has proposed that reduced cognitive efficiency might occur as a result of changes in the temporal dynamics of DLPFC recruitment. In this study, we used a mixed blocked/ event-related fMRI design to track transient and sustained activity in DLPFC while high- and low-anxious participants performed a working memory task. The task was performed after the participants viewed videos designed to induce neutral or anxiety-related moods. After the neutral video, the high-anxious participants had reduced sustained but increased transient activation in working memory areas, in comparison with low-anxious participants. The high-anxious group also showed extensive reductions in sustained activation of "default-network" areas (possible deactivation). After the negative video, the low-anxiety group shifted their activation dynamics in cognitive control regions to resemble those of the high-anxious group. These results suggest that reduced cognitive control in anxiety might be due to a transient, rather than sustained, pattern of working memory recruitment. Supplementary information for this study may be found at www.psychonomic.org/archive.
\end{abstract}

Anxiety is a negative emotion frequently accompanied by changes in cognitive processing. The behavioral expression of these changes has been extensively studied, but their neural substrate is not well understood. Behaviorally, abnormalities in attentional control are often seen when subjects are presented with threat-related stimuli or distractors thought to be anxiety inducing (Fox \& Georgiou, 2005; Koster, Crombez, Verschuere, Van Damme, \& Wiersema, 2006). In addition, individuals who are temperamentally anxious show impairments in cognitive tasks that lack any explicit threat-related material. For example, there is evidence that trait anxiety reduces working memory (Derakshan \& Eysenck, 1998; Leon \& Revelle, 1985; Richards, French, Keogh, \& Carter, 2000; Tohill \& Holyoak, 2000), as well as complex reasoning (e.g., Darke, 1988; Derakshan
\& Eysenck, 1998; Richards et al., 2000; Zarantonello, Slaymaker, Johnson, \& Petzel, 1984). This research suggests that trait or state anxiety can modulate cognitive control even when the stimuli themselves are not anxiety inducing. Moreover, in cognitive tasks without affectively valenced stimuli, impairments are found with difficult tasks, but not with easy ones. Several studies have manipulated task difficulty and working memory load, reporting anxiety deficits only at the more difficult levels (Calvo, 1985; Calvo \& Ramos, 1989; Calvo, Ramos, \& Estevez, 1992; Eysenck, 1982, 1985; Eysenck \& Calvo, 1992; Hamilton, 1978).

\section{Anxiety and Processing Efficiency}

Results such as these have led some researchers to suggest that anxiety does not affect cognitive abilities directly, 
but rather the efficiency with which cognitive tasks are performed. To explain inconsistent findings in the literature on anxiety and cognitive function, Eysenck and Calvo (1992; Eysenck, Derakshan, Santos, \& Calvo, 2007) proposed a processing efficiency theory. According to this theory, anxious arousal may lead to worry and ruminative thoughts intended to dispel dysphoric feelings (Borkovec \& Roemer, 1995). This threat-related thinking is believed to intrude on normal cognition by taking up valuable working memory resources. Additionally, anxiety-related autonomic activity (in response to anxiety-inducing task stimuli) may further impair cognitive control by distracting anxious individuals from focusing on the task at hand. However, a high level of motivation to reduce the aversive state may lead to a compensatory enhancement of cognitive effort, in order to maintain a standard level of performance. Such enhanced effort could be associated with increased activation in brain regions associated with cognitive control, such as dorsolateral or ventrolateral prefrontal cortex (DLPFC or VLPFC) or dorsal anterior cingulate (ACC; Cazalis et al., 2003; Donohue, Wendelken, \& Bunge, 2008; Wagner, Maril, Bjork, \& Schacter, 2001). If so, this pattern would be consistent with neuroimaging studies that interpret relatively greater activation in cognitive control areas (given equal performance) as a sign of reduced processing efficiency (Gray et al., 2005). However, anxiety has also been associated with underactivation in cognitive control neural circuits (Bishop, 2007; Bishop, Duncan, Brett, \& Lawrence, 2004), a finding which has also been interpreted as deficient recruitment of cognitive control. Thus, simply examining over- or underutilization of a neural area may not be optimally informative about cognitive abilities in anxious subjects. Either reduced or enhanced neural recruitment may reflect differences in efficiency, motivation, or effort, or in the capacity to activate regions when needed; or in some combination of all of these.

\section{Dual Mechanisms of Cognitive Control}

A different approach to cognitive efficiency stresses the dynamics of cognitive control recruitment, suggesting that cognitive control can be exerted in either a sustained or a transient fashion, depending on the task context or demands. This theory, termed dual mechanisms of control (Braver, Gray, \& Burgess, 2007), postulates that there are really two types of cognitive control, corresponding to when control mechanisms are exerted. Proactive control, emphasizing early selection of attentional focus, would be exemplified by a sustained representation of task requirements or goals throughout periods of high control demand. This sustained task representation would allow for more effective top-down control of processing: It is considered to promote preparatory attentional and response biases and the prevention (or resolution) of conflict during ongoing processing. By contrast, reactive control would have an only-when-needed "late correction" character. In reactive mode, when task goals are first encoded, they may be represented only transiently in working memory. After that, they may not be maintained in a continuously active state. With reactive control, task representations are reactivated only as needed (that is, when a task-relevant stimulus is encountered, or conflict occurs in processing). Reactive control is thus thought to entail weaker preparatory attentional biases, and processing is therefore more easily influenced by bottom-up input. As such, this type of control would be more desirable when unpredictable threats are possible in the environment, or when environmental monitoring or responsivity to changing task contingencies are needed. In such cases, top-down control of attention could be a liability, since a bias to perceive only specific kinds of stimuli would leave the organism slower to respond to unexpected threats. The DMC theory posits that these two forms of cognitive control are used by virtually all people at different times. However, Braver et al. also speculated that although proactive control may be more effective in performing certain types of cognitive tasks, anxious people may be more prone to reactive control in many situations as an adaptation to the presence of anxiety arousal and the need for environmental vigilance. In neuroimaging terms, this would suggest that high-anxiety states or traits should be linked to greater transient activation, but less sustained activation, in areas such as DLPFC or other cognitive control regions.

\section{Anxiety-Cognition Interaction in the Brain}

A small number of studies have examined the effects of anxiety on cognitive control areas during performance of cognitively demanding tasks. One group (Eisenberger, Lieberman, \& Satpute, 2005) found that dorsal ACC activity increases with trait neuroticism, and another (Dolcos \& McCarthy, 2006) showed that lateral PFC activity decreases with threat distractors. Bishop et al. (2004) found reduced recruitment of DLPFC in higher-anxiety participants as they encountered cues that predicted frequent threat-related distractors in an attentional task. Although informative, these studies were not designed to dissociate transient and sustained response to the tasks.

A second route by which anxiety might influence cognitive control and the associated brain regions is by modulating regions involved in emotional processing, chiefly ventromedial PFC (Simpson, Snyder, Gusnard, \& Raichle, 2001) and the amygdala (Dolcos \& McCarthy, 2006). In a comprehensive review of anxiety effects on attentional control, Bishop (2007) has suggested that, in comparison with nonanxious individuals, people experiencing state anxiety show hyperresponsive amygdala activity in the presence of threat-related distractors, and that trait-anxious individuals, in particular, are also likely to show reduced regulatory response in lateral prefrontal cortex. Ventromedial PFC regions have usually been included in the "default-mode" system, a network of regions (Shulman et al., 1997) whose activity is closely, but inversely, related to processing in cognitive control areas. The default network (consisting of medial PFC, left lateral inferior PFC, posterior cingulate/precuneus, lateral parietal cortex, left inferior temporal lobe, and sometimes the amygdala) has been proposed to support a broad spectrum of cognitively undemanding functions, such as environmental or visuospatial monitoring, social and self-referential processing, emotion processing and emo- 
tional memory, autobiographical memory, and mediation of resting-state mental activity (Drevets, 2001; Greicius, Krasnow, Reiss, \& Menon, 2003; Gusnard \& Raichle, 2001; Maddock, 1999; Maddock, Garrett, \& Buonocore, 2003; Mazoyer et al., 2001). More importantly, default regions have been characterized as a "network" precisely because they tend to collectively grow less active (possibly reflecting active suppression) just when cognitive control areas become more active (Drevets \& Raichle, 1998; Shulman et al., 1997). In particular, default areas tend to show decreasing activation as cognitive tasks become more difficult (Greicius et al., 2003; McKiernan, Kaufman, Kucera-Thompson, \& Binder, 2003). More recently, Weissman and colleagues (Weissman, Roberts, Visscher, \& Woldorff, 2006) have reported a loss of deactivation of default-network regions for trials in which response times (RTs) suggest momentary lapses in attention. These studies are consistent with the hypothesis that reduced activation in default areas plays a role in efficient cognitive processing, and that performance might in fact depend on this reduction. Importantly, default-network regions (especially ventromedial PFC) most commonly show increased activation (reduced deactivation) in the presence of anxiety (trait or state) when no cognitive task is required (Simpson, Drevets, Snyder, Gusnard, \& Raichle, 2001; Zald, Mattson, \& Pardo, 2002). Emotional modulation of default regions thus may prevent the decreased activation needed for optimal cognitive efficiency, which might in turn impair or even directly suppress activity in cognitivecontrol regions. However, Simpson and colleagues (Simpson, Drevets, et al., 2001; Zald et al., 2002) also found that individuals with lower trait anxiety responded to an anxiety induction by reducing activation in ventromedial PFC. This suggests that in some situations, state or trait anxiety could lead to a compensatory strategy of reduced tonic activation of default regions, especially during difficult cognitive tasks. Such suppression might take place in order to improve performance by limiting the potential for interfering emotional thoughts to arise.

\section{The Present Study}

The research reviewed above suggests that both the cognitive-control and default brain networks could play a role in the effects of anxiety on cognitive performance. To examine this question in the present study, we used a hybrid fMRI design (blocked/event-related) to examine sustained and transient activity in a priori-defined regions in cognitive-control and default-network circuitry. Participants were asked to perform demanding cognitive tasks (verbal working memory) that provided three trial types of varying difficulty. We first examined responses in brain areas as a function of individual differences in trait anxiety during an affectively neutral mood condition. Second, we used affectively valenced videos as a mood-manipulation technique (Gray, 2001; Gray, Braver, \& Raichle, 2002) to induce state anxiety associated with a negative mood condition.

We asked the following three questions.

1. Would trait anxiety in a neutral mood modulate the level of sustained activity in cognitive-control or default- network areas? We predicted that trait anxiety might be associated with reduced sustained activation in cognitive control areas if anxious individuals were less able to maintain an ongoing attentional focus. We also hypothesized that trait anxiety might be associated with increases in sustained activation in the default network (especially emotion-processing areas such as ventromedial PFC).

2 . Would trait anxiety in a neutral mood modulate the level of transient activity in cognitive-control or defaultnetwork areas? We predicted that anxiety would modulate transient responses generally across all trial types, but also show additional trial-type-specific effects. Across all trial types, we expected that trait-anxious individuals would show greater transient activity in cognitive control areas if they had to compensate for reduced sustained activation. For individual trial types, we predicted that trait-anxious participants would have difficulty transiently reducing activation (deactivating) in default-mode regions in response to the more difficult trial types. In addition, we expected that we might also see alterations in recruitment of cognitive control regions for the more difficult trials.

3. Would a negative mood state after an anxietyinducing video modulate sustained or transient activity? We reasoned that either sustained or transient responses might show such modulation, and that induced state anxiety could also have either of two general effects on the two groups. First, an increase in negative mood could have a main effect across the two groups, leading both to increase anxiety-related recruitment patterns; that is, a negative mood might induce low-anxiety participants to activate brain regions in a manner similar to those seen in highanxiety participants in a neutral mood, but exacerbate, in the high-anxious group, the differences already seen in the neutral condition. Alternatively, the negative mood induction might interact with trait anxiety, in that the lowanxious group would increase signs of anxiety-related processing (reduced sustained and increased transient recruitment of cognitive control regions), whereas the highanxiety participants would not change (e.g., if they were already at asymptotic anxiety levels at baseline).

\section{METHOD}

\section{Participants}

A total of 102 participants (Washington University students and individuals from the surrounding community), screened for neurological disorders and use of psychoactive drugs, took part in the study (Table 1) after giving informed consent. Of participants completing all tests, 6 were eliminated because of a significantly depressed mood (score greater than 25 on the Beck Depression Inventory [BDI; Beck, Ward, Mendelson, Mock, \& Erbaugh, 1961]), poor performance on the behavioral task, or insufficient numbers of trials for one of the conditions. Of the remaining participants $(n=$ 96,41 males and 55 females), mean age 22.2 years $(S D=4.9)$, no participant was older than 36 . All participants were right-handed. An overall anxiety score was computed for each of the 96 participants on the basis of their responses to assessments of anxiety and worry (see below). These scores were then used to select participants in the highest and lowest $20 \%$ (approximate) groups with respect to anxiety scores (high-anxiety, $n=20$; low-anxiety, $n=20$ ). There were 11 males and 9 females in the low-anxiety group and 3 males and 17 females in the high-anxiety group. Participants were paid 
Table 1

Participant Demographics: Mean Values (and Standard Deviations) for the Patient and Control Groups

\begin{tabular}{|c|c|c|c|c|c|c|c|c|c|c|c|}
\hline \multirow[b]{2}{*}{ Group } & \multirow[b]{2}{*}{$N$} & \multicolumn{2}{|c|}{ Age } & \multicolumn{2}{|c|}{ Gender } & \multicolumn{2}{|c|}{ TMAS } & \multicolumn{2}{|c|}{ PSWQ } & \multicolumn{2}{|c|}{ BDI } \\
\hline & & $M$ & $S D$ & M & $\mathrm{F}$ & $M$ & $S D$ & $M$ & $S D$ & $M$ & $S D$ \\
\hline All & 96 & 22.2 & \pm 4.9 & 44 & 51 & 6.3 & \pm 3.9 & 41.7 & \pm 11.8 & 7.2 & \pm 5.6 \\
\hline Low & 20 & 22.3 & \pm 5.0 & 11 & 9 & 2.8 & \pm 1.1 & 28.0 & \pm 6.0 & 5.8 & \pm 5.7 \\
\hline High & 20 & 20.5 & \pm 1.9 & 3 & 17 & 11.2 & \pm 2.6 & 55.3 & \pm 10.2 & 12.1 & \pm 6.8 \\
\hline
\end{tabular}

Note-TMAS, Taylor Manifest Anxiety Scale; PSWQ, Penn State Worry Questionnaire; BDI, Beck Depression Inventory.

$\$ 10 / \mathrm{h}$ for personality and neuropsychological testing, and $\$ 25 / \mathrm{h}$ for the scanning session.

\section{Materials and Procedure}

The data presented here were collected as part of a larger study on cognitive and affective individual differences. Testing and assessments were administered in two separate sessions, spaced a few days to a few weeks apart. In the first session, participants completed personality and mood questionnaires, then took neuropsychological tests. The second session consisted of functional neuroimaging during cognitive task performance, with each scan preceded by a video viewing to manipulate mood.

Personality and mood assessments. In all, 20 personality assessment tests were administered. The present study focuses on a subset of these, measuring anxiety and generalized negative affect. Trait measures of anxiety and ruminative worry were measured using the Taylor Manifest Anxiety Scale (TMAS; Taylor, 1953) and the Penn State Worry Questionnaire (PSWQ; Brown, Antony, \& Barlow, 1992; Meyer, Miller, Metzger, \& Borkovec, 1990). Mood state for the prior 1 week was assessed using the BDI (see the online supplementary information, available at www.psyconomic.org/archive, for details of these assessment tools).

Mood manipulation. Our previous research (Gray, 2001; Gray et al., 2002) has validated that presentation of short videos can induce predictable mood states. In this study, we used videos of approximately $10 \mathrm{~min}$ each that were intended to induce either a negative or a neutral mood. (BOLD data was not collected during the video presentation.) The negative videos were selected to be anxiety-provoking, and included scenes from horror movies (fixed clips from Scream and Halloween), whereas the neutral videos included documentaries (fixed clips from Michael Moore's Roger and Me and from a film about vacationing in Australia). (During the scanning session, participants also viewed amusing videos prior to other scans; these scans were not the focus of the present study and will not be discussed further.) The two videos for a single valence were always presented in sequential pairs to promote more consistent mood manipulation over time. This yielded video sequences in the pattern: AA-BB-CC, with the order of the three valences counterbalanced across participants. The videos were projected onto a screen at the head of the bore that was visible to the participant by use of a mirror attached to the head coil, with audio content via headphones.

After each scanning run, we asked participants to rate their mood state using Profile of Moods State (POMS) tests, in order to check on the effectiveness of the mood manipulation. In the present study, participants rated their moods retroactively, reporting on their memory of how they felt immediately after viewing each video. Ratings after the neutral videos were used as an estimate of participants' baseline affective state.

The $\boldsymbol{n}$-back task. The cognitive task used was a verbal threeback task. This is a demanding working memory task that requires participants to say, on any given trial, whether the word currently displayed is the same as or different from the word displayed three words back (Braver et al., 1997; Gray, Chabris, \& Braver, 2003). Thus, participants must keep a running tally of the last three words displayed, and must update this list after each response. The task was implemented using PsyScope software (Cohen, MacWhinney,
Flatt, \& Provost, 1993) and run on a Macintosh G3 processor connected to a video screen in the scanner. Each word was presented for $2 \mathrm{sec}$, followed by a 360-msec fixation cross. Participants gave their responses by pressing "same" or "different" buttons on a fiber-optic light-sensitive keypad that was connected to the PsyScope button box. This task can be broken down into three types of trials: "targets," "lures," and "nonlures" (Gray et al., 2003). Target trials were those in which the correct answer was "same," because the word matched the word three trials back. Lure trials were nontargets ("different" from the word three back), but they presented a word that had been presented at least once previously in the current block. Nonlure trials were also "different," but the word presented was being shown for the first time. (See the supplementary information for details on cognitive interpretation of these trial types.)

Scanning procedure. At the beginning of a scanning session, participants were first given instructions and practice on performing the three-back task. Practice blocks could be repeated if necessary. The scanning session included six scans, with each involving presentation of a mood-inducing video, followed immediately by three-back task performance. Scanning was conducted only during three-back performance and not during video presentation. Three of the scans involved $n$-back task performance with neutral words as stimuli; the other three had neutral face stimuli. For the present study, we report on results from two of the scans involving word stimuli, one with a neutral video and the other with an anxiety-related video. ${ }^{1}$ After performance of the three-back task, participants were asked to report on what their mood state had been immediately following the video (before they performed the three-back task), using a computerized version of the POMS assessment.

In order to estimate blocked and trial-related neuroimaging effects separately, we used a mixed design (Donaldson, Petersen, Ollinger, \& Buckner, 2001; Visscher et al., 2003). The mixed design calls for blocks of task trials that alternate with blocks of rest. Within each task block, trials are presented in an event-related manner, with variable-length intervals between trials. Trial-specific effects are then estimated as the transient activity locked to onset of a trial (or trial event). Sustained task-related effects are estimated (while transient effects are simultaneously controlled for, using multiple regression) as the contrast of activity within the task block versus activity during rest blocks.

In the present study, each functional run consisted of three fixation (F) blocks (15 trials duration) alternating with two task (T) blocks (32 trials per block), in an F-T-F-T-F sequence. Within each task block, stimulus words were presented for $2 \mathrm{sec}$ each, followed by a 0.36 -sec fixation cross. Responses had to be given within the 2-sec stimulus presentation interval, so that a single TR $(2.36 \mathrm{sec})$ covered the entire trial. Intertrial intervals (ITIs) were of variable length $(0,2,360$, and $4,720 \mathrm{msec}$ long, distributed exponentially, for an average ITI length of $1,530 \mathrm{msec}$ ). Each functional run acquired 149 volume images, beginning with 4 that were not analyzed. The three fixation blocks consisted of 15 frames each (a black screen with fixation cross in white), and the two task blocks consisted of 50 frames each: 32 trials and 18 intertrial jittered fixations.

Within each run, a list of 28 words - all nonemotional, concrete nouns - was presented in fixed order over the 64 trials. Six different 
word lists were possible, and their order was counterbalanced across participants. Approximately $31 \%$ of trials were targets, $50 \%$ were nonlures, and $19 \%$ were lure trials. After the end of the run, scanning ceased, and participants were asked to rate their mood states, using POMS tests. Specifically, they were asked to think back and report on what their mood had been immediately after the video (i.e., before they began the three-back trials).

Image acquisition. fMRI images were collected on a Siemens 3T Allegra MRI scanner (Erlangen, Germany). The protocol included localizer images, low- and high-resolution structural images, a T2-weighted image, and a series of functional images. The structural images were acquired with $1 \times 1 \times 1.25$ resolution, using a sagittal 3-D T1-weighted sequence. Functional images were collected using an asymmetric spin-echo echo-planar sequence with volume $\mathrm{TR}=2.36 \mathrm{sec}, \mathrm{TE}=50 \mathrm{msec}$, and flip angle $=90^{\circ} . \mathrm{A}$ volume image consisted of 32 transverse slices, $4 \mathrm{~mm}$ thick, with an in-plane resolution of $3.75 \times 3.75 \mathrm{~mm}$.

Image preprocessing. The functional imaging data were preprocessed to correct for asynchronous slice acquisition and odd-even slice intensity differences caused by interleaving. Following this, the data were normalized to a fixed image intensity value within each scanning run; then, rigid body motion was corrected (Friston, Williams, Howard, Frackowiak, \& Turner, 1996; Snyder, 1996). Atlas transformation (12 parameter affine) of the functional data was computed via the structural images. Our atlas-representative target image conforms to the space of Talairach and Tournoux (1988) as defined by Lancaster et al. (1995). The final preprocessing step combined motion correction and atlas transformation in one resampling to 3-mm isotropic voxels. Before statistical analysis, the data were smoothed using a Gaussian filter with 9-mm full width at half maximum.

The preprocessed data were analyzed using the general linear model (GLM), coding several regressors for transient effects, a single regressor for sustained effects, and two regressors for the baseline signal and the slope of linear drift, all modeled simultaneously. Sustained effects were modeled by a single regressor defined to have the shape of a gamma function convolved with a boxcar function. This effect was estimated as any systematic variance in the signal that was not accounted for by transient effects. For the sustained effect, a single magnitude was calculated for each voxel for the entire length of a task block (for each of two task blocks per run, and excluding the fixation blocks). Transient effects were estimated using eight time-point regressors (starting at trial onset), for each of three trial types (targets, nontarget "lures," and nontarget "nonlures"). Total time-course length was therefore $18.88 \mathrm{sec}$. Analyses of transient trial-type responses used magnitude estimates, defined as the cross-correlation of the estimated BOLD response over the eight time-points for the trial condition with a canonical hemodynamic response function (HRF). The HRF was modeled as a gamma function with a delay of $2 \mathrm{sec}$ and a time constant of $1.25 \mathrm{sec}$ (Boynton, Engel, Glover, \& Heeger, 1996). Combined item effects were calculated by summing across all trial types (for the two scans of interest in this study). Except where otherwise noted, all effects are given in terms of percent signal change, calculated as the signal magnitude divided by mean estimate of the baseline signal.

\section{fMRI Analysis}

We used in-house software to carry out correlations, ANOVAs, or $t$ tests to identify regions significantly activated (or deactivated) for each of several effects. We arrived at an overall measure of transient effects by averaging activation across all trial types (correct trials only).

Region-of-interest (ROI) selection. For the present study, we restricted our analyses to brain regions belonging to working memory or default-network regions. We created a mask of a priori ROIs for working memory areas (see Figure 1 and Table A1 in the Appendix), using neuroanatomical coordinates described in a metaanalysis of $n$-back neuroimaging results (Owen, McMillan, Laird, \& Bullmore, 2005), and of working memory tasks more generally (Wager \& Smith, 2003). We omitted coordinates for subcortical locations, creating spherical ROIs of $20 \mathrm{~mm}$ in diameter for cortical areas. (See the supplementary information for details.) To create a mask of a priori default-network regions, we used an activation map (see Figure 1) described in Shulman et al. (1997) and Buckner et al. (2005), which enabled the use of full regions, rather than being defined on the basis of centroid (see the Appendix). We constrained the extent of the default regions by thresholding the map at 16 PET "counts"2 (representing a 1.6\% change in regional cerebral blood flow relative to the whole brain mean), as originally described by Shulman et al.

fMRI data analysis. In this article, we took two approaches to analyze the data. As an overview of network-level results, we first created two "composite-network ROI masks" (one each for the cognitive-control and default networks) by combining all the sepa-

Cognitive Control Regions

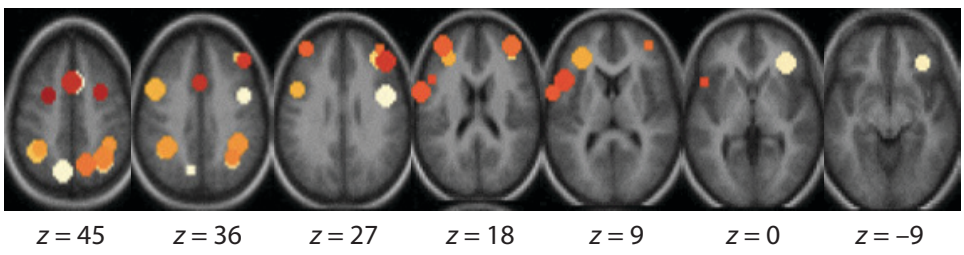

Default-Network Regions

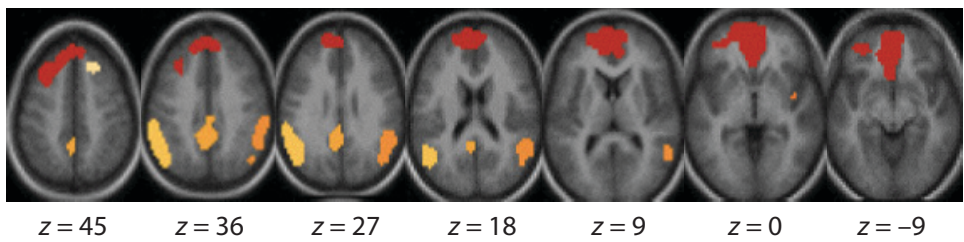

Figure 1. ROI masks used for identifying cognitive control and default-mode regions. Cognitive control regions include $20-\mathrm{mm}$ spheres centered at loci taken from two meta-analyses of working memory and verbal $\boldsymbol{n}$-back performance. Default-network regions include regions known to show task-related deactivation. (See the Method section for details. The Appendix provides specifics on the individual regions.) 
rate regions in each network (see Figure 1) into single regions of interest. Each composite-network mask thus consisted of a single large ROI covering all the regions of interest in that network. We then averaged activation across voxels falling anywhere within the mask. This analysis, which provides a robust and powerful test of our hypotheses within a single analysis, is reported in this article.

Second, to determine individual regions of activity within each network, we conducted a more conventional analysis, detailed in the supplementary information, that identified voxels falling within our two a priori masks, and also showing significant task-related activity for the effects of interest. Voxels thus identified were clustered and subjected to region-wide analyses; regions showing significant group differences are summarized in Table A2.

\section{Behavioral Analysis}

We conducted correlational analyses to test for any association between anxiety and performance on the three-back task, in either the neutral or negative-mood condition. In addition, we looked for group differences in performance (between the high- and low-anxiety groups) using independent $t$ tests or repeated measures ANOVAs on RT and accuracy, with a within-participants factor of trial type.

\section{RESULTS}

\section{Behavioral Results}

Anxiety and other personality measures. The TMAS scores for all 96 participants had a mean value of 6.33 $(S D=3.4)$, with range 1 to 16 . The PSWQ scores had a mean value of $41.7(S D=11.8)$, with range 16 to 78 . These two measures, which were significantly positively correlated $(r=+.58, p=.000)$, were normalized as $z$-scores and combined to produce composite trait-anxiety scores. (See the online supplementary information for details on how results for the composite trait-anxiety scores differed from results for the PSWQ and TMAS scores separately.) The range of trait-anxiety $z$-scores for all 96 participants was -2.72 to 4.43 . Low-anxious participants had a mean anxiety $z$-score of $-1.97(S D=0.44)$ and range of -2.72 to -1.35 . High-anxious participants had a mean anxiety $z$-score of $2.25(S D=1.0)$ and range of 1.27 to 4.43 . The high-anxious group was significantly more depressed (BDI scores: $M=12.1, S D=6.8$ ) than the low-anxious group (BDI scores: $M=5.8, S D=5.7$ ) $[t(38)=-3.20, p=.003$, $\left.\eta^{2}=.21\right]$. In addition, the high-anxious group was disproportionately female ( 3 males, 17 females), whereas the low-anxious group was more mixed (11 males, 9 females), resulting in a statistically significant association between gender and anxiety group (Fisher's exact test [two sided], $p=.019)$. The two groups did not differ by age $(p>.1)$.

Mood-manipulation check. In this article, we report only on the POMS-anxious and POMS-gloomy scores to indicate the effects of the videos. For the full sample of 96 participants, the POMS-anxious scores following the negative movie were significantly greater than those for the neutral movie $\left[t(94)=7.77, p<.001, \eta^{2}=.39\right]$, suggesting that the videos did induce a more anxious mood. We also conducted an ANOVA on the POMS-anxious scores for just the subsample that included the high- and low-anxiety groups, with video type (neutral vs. negative) and group as factors. There was again a main effect of video type, with anxiety after the negative video significantly greater than after the neutral movie $\left[F(1,38)=32.68, p<.001, \eta^{2}=\right.$ .46], as well as a trend toward a significant effect of group
$\left[F(1,38)=3.72, p=.061, \eta^{2}=.09\right]$, with the anxious group scoring higher in anxiety. However, the interaction between group and video type was nonsignificant $(p>.1)$. In addition, the effect of the video manipulation on the POMS-anxious score did not interact with gender, either alone or when trait anxiety ratings were covaried out.

The POMS-gloomy scores in the full sample were also significantly higher after the negative video than after the neutral video $\left[t(94)=2.67, p=.009, \eta^{2}=.07\right]$, indicating that the movie clips also induced a concurrent gloomy or depressed mood. However, a two-way ANOVA restricted to just the high- and low-anxiety groups (group $X$ video type) found that the main effect of video type did not quite reach significance $(p=.11)$, nor did the interaction between group and video type $(p>.8)$. There was a main effect of group $\left[F(1,38)=5.60, p=.023, \eta^{2}=\right.$ $.13]$, confirming the BDI measure showing that the highanxious group was significantly more depressed than the low-anxious group, independent of the video-type manipulation. Finally, the effect of the video manipulation on the POMS-gloomy score did not interact with gender, either alone or when trait anxiety ratings were covaried out.

\section{Three-Back Task Performance}

$\boldsymbol{n}=$ 96. ANOVAs and correlational analyses were conducted to examine behavioral performance for all 96 participants. Results were not different from those found for the high- and low-anxious groups. For details on these analyses, see the supplementary information.

$\boldsymbol{n}=\mathbf{2 0}$ per group. The general trial type effects on three-back performance found in the full sample were replicated in the subgroups (see Figure 2). A two-way ANOVA (video type $\times$ trial type, omitting the group factor) on percent correct revealed a main effect of trial type $\left[F(2,38)=70.29, p<.001, \eta^{2}=.79\right]$. Lure trials were significantly less accurate than targets $[F(1,39)=5.18$, $\left.p=.028, \eta^{2}=.18\right]$ and they were also significantly less accurate than nonlures $\left[F(1,39)=91.76, p<.001, \eta^{2}=\right.$ .70]. Target trials were also significantly less accurate than nonlures $\left[F(1,39)=121.40, p<.001, \eta^{2}=.76\right]$. However, there was no main effect of video type or video type $\times$ trial type interaction on accuracy (both $p s>.4$ ).

A similar pattern was found in RT, a robust main effect of trial type $\left[F(2,38)=47.68, p<.001, \eta^{2}=.72\right]$, but no main effect or interaction with video type (all $p \mathrm{~s}>.5$ ). Again, lure trials were significantly slower than targets $\left[F(1,39)=34.52, p<.001, \eta^{2}=.47\right]$, and they were also significantly slower than nonlures $[F(1,39)=93.73, p<$ $\left..001, \eta^{2}=.71\right]$. Targets were also significantly slower than nonlures $\left[F(1,39)=8.33, p=.006, \eta^{2}=.18\right]$.

Including the group factor, we ran a three-way repeated measures ANOVA (video type $\times$ trial type $\times$ anxiety group) and found no significant main effect of group on $\mathrm{RT}$ or accuracy (all $p \mathrm{~s}>$.2). Interactions with group were also nonsignificant (all $p \mathrm{~s}>.2$ ).

\section{Neuroimaging Results}

In this study, regions showing significant brain activity were identified as a function of four different factors: network (cognitive control or default), signal type (sustained 


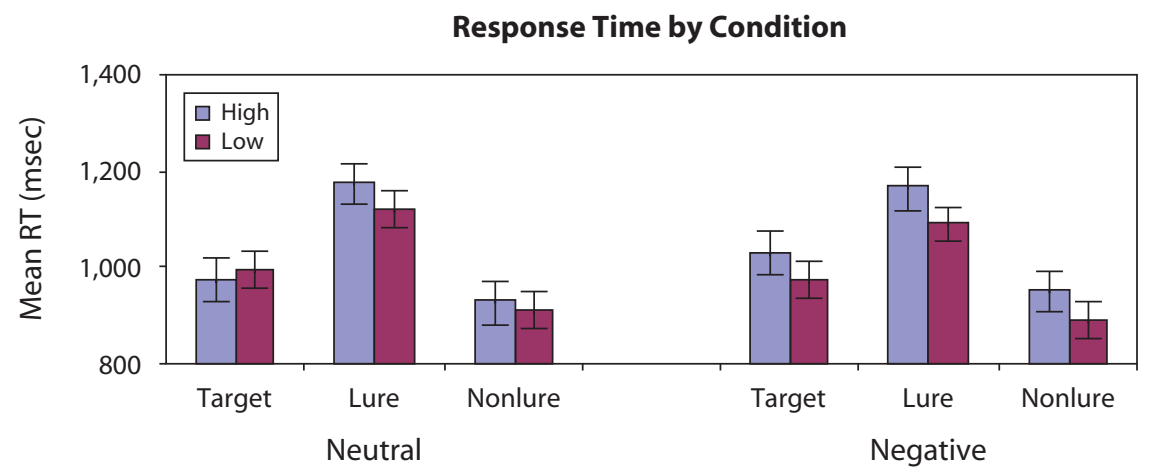

Accuracy by Condition

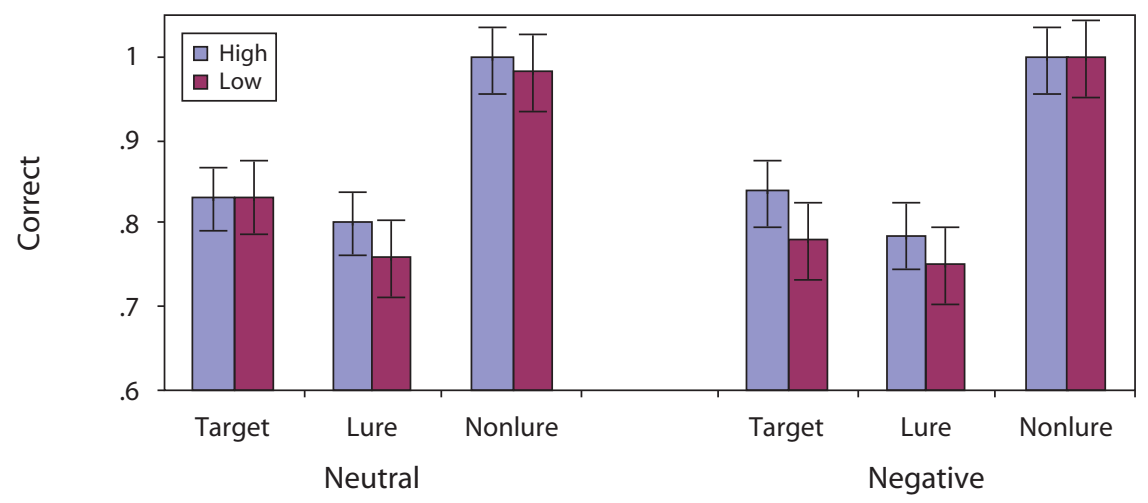

Figure 2. Behavioral performance in the three-back task. Error bars show standard errors of the means.

or transient), video (neutral or negative), and group (low or high anxiety). A fifth factor, trial type (target, lure, and nonlure), was used to investigate trial-specific effects in the neutral condition only. In this article we describe the effects of anxiety on sustained and transient activity in each network as a whole, without regard to individual regions. Results at the level of individual regions did not diverge from results presented for the networks as a whole. Thus, findings for individual regions are summarized in Table A2 (also see Figure 4), but the corresponding analyses are detailed in the supplementary information. (An exception is the description of trial-type effects, which are presented for individual regions, and are described fully in this article.) We report all results in terms of increased or reduced activation (signal magnitude). When activation levels for default-network regions were reduced significantly below baseline, we have also noted this as possible deactivation, although we take no position as to whether a negative-going signal represents reduced activation or an active inhibitory process.

\section{Neutral Condition}

Anxiety effects on sustained and transient response. A three-way ANOVA (network $\times$ signal $\times$ group) on the data from the neutral condition revealed a significant two-way interaction between signal and group $\left[F(1,38)=11.74, p=.001, \eta^{2}=.24\right]$. As is shown in Figure 3 , the high-anxious group showed less sustained activation than did the low-anxious group in both networks [cognitive-control, $t(38)=1.99, p=.05, \eta^{2}=$ .09 ; default, $\left.t(38)=2.25, p=.03, \eta^{2}=.12\right]$. By contrast, the high-anxious showed significantly greater transient activation in the cognitive-control network than did the low-anxious $\left[t(38)=-2.73, p=.010, \eta^{2}=.16\right]$ and no significant difference in the default network $(p>.2)$. The three-way interaction of network $\times$ signal $\times$ group was not significant $(p>.4)$. (Figure 4 shows individual regions where significant group differences in activation were found.)

Generality of anxiety effects across whole sample $(n=96)$. To test the robustness of our group difference effects in a continuous sample, we examined correlations between anxiety (across the full sample of 96 participants) and sustained or transient activity in the two networks. Sustained activation in the cognitive-control network showed a significant inverse correlation with anxiety score $(r=-.233, p=.023)$, whereas transient cognitivecontrol activation was significantly positively correlated with anxiety $(r=+.345, p=.001)$. By contrast, sustained activation in the default network showed a trend toward a significant inverse correlation with anxiety $(r=$ $-.196, p=.056)$, whereas transient default activation was, significantly, positively correlated with anxiety $(r=$ $+.213, p=.037$ ). Thus, across both networks, continuous variation in trait anxiety (across all 96 participants) was negatively related to the sustained portion of the BOLD 

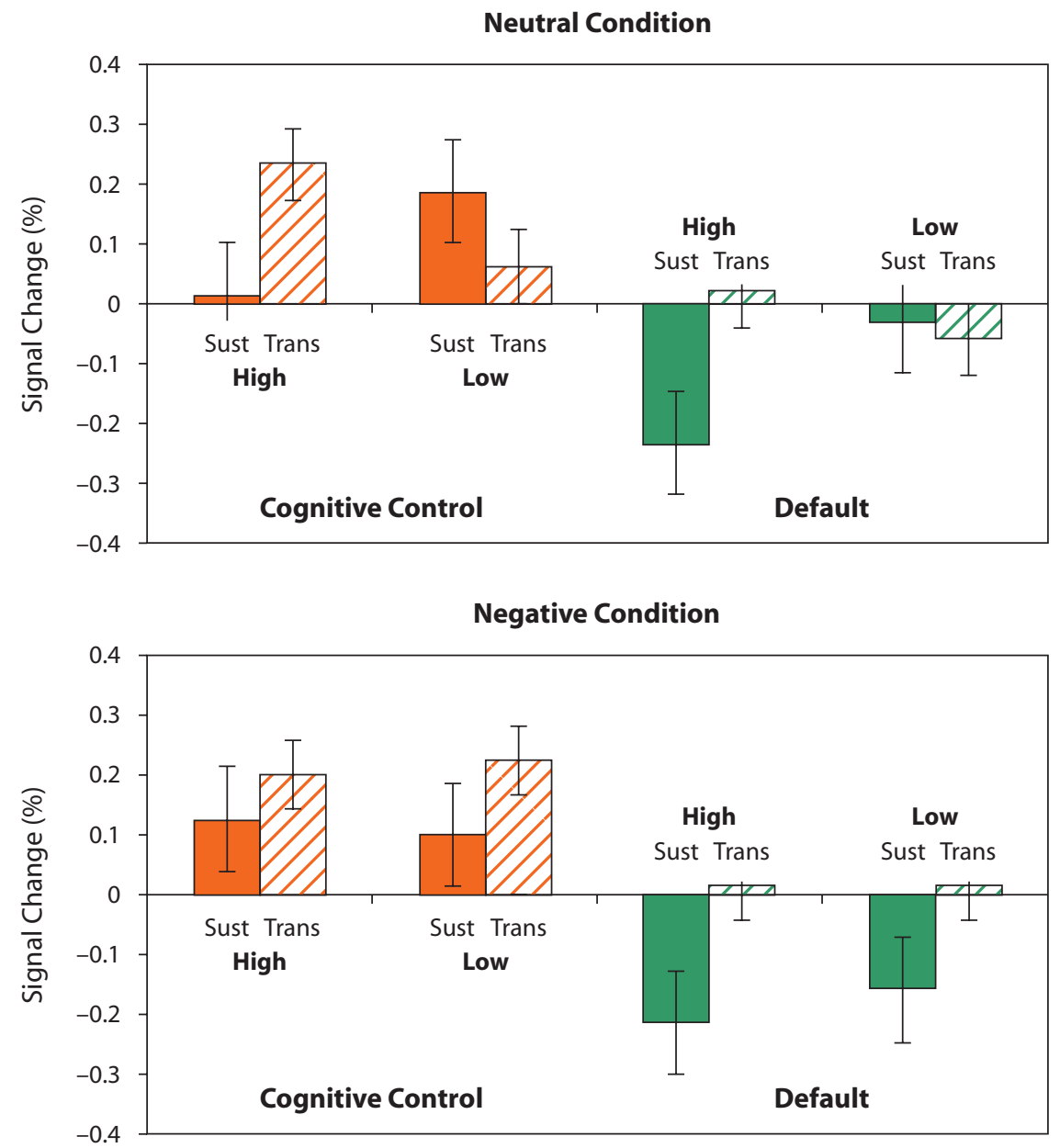

Figure 3. Network-wide activation averaged across composite-network ROIs (cognitive control or default network). Neural activity is shown for each group by signal type (sustained or transient), in the neutral condition (top panel) and in the negative condition (bottom panel).

response, and positively related to the transient portion. Correlation coefficients for the individual regions in each network mirror this pattern and are listed in Table A2. (See the supplementary information for details.)

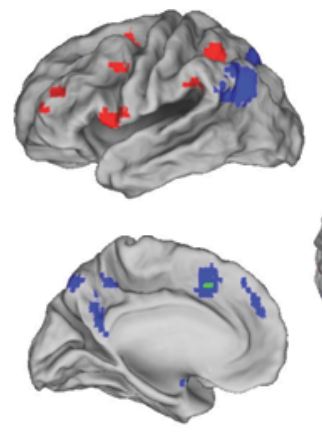

Left Hemisphere

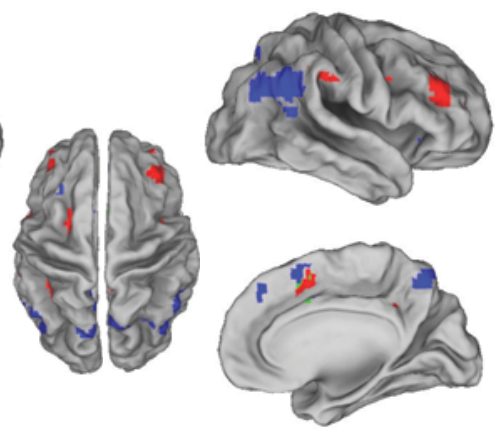

Right Hemisphere
Figure 4. Areas of significant group differences in sustained activation (blue regions), in transient activation (red regions), and overlapping sustained/transient activation (green regions).
Trial-type effects in individual regions. To investigate the source of possible differences in cognitive efficiency, we conducted a repeated measures ANOVA with trial type (targets, lures, and nonlures) and group as factors. (Regions showing a significant trial type $\times$ group interaction were identified as described for transient responses in the supplementary information.) We found only two regions that showed a significant trial type $x$ group interaction (see Figure 5). These were a cognitivecontrol region in right inferior frontal cortex (Brodmann area [BA] 47, with center at Talairach coordinates 33, 28, $-3)$ and a default-mode region in the left inferior parietal lobe (IPL; BA 40, in the supramarginal gyrus, with coordinates $-57,-47,35)$. In the right $\mathrm{BA} 47$ region, the highanxious group showed an effect of trial type due to transient activation for lure trials that was significantly higher than for targets or for nonlures. The low-anxious group showed no significant effect of trial type. In contrast, the effect of trial type in the left IPL was due to significantly greater reductions in activation (greater deactivation) in the low-anxious group for lure trials than for targets or nonlures. The high-anxious group showed a trend to a trial 
Right Ventrolateral PFC (BA 47)
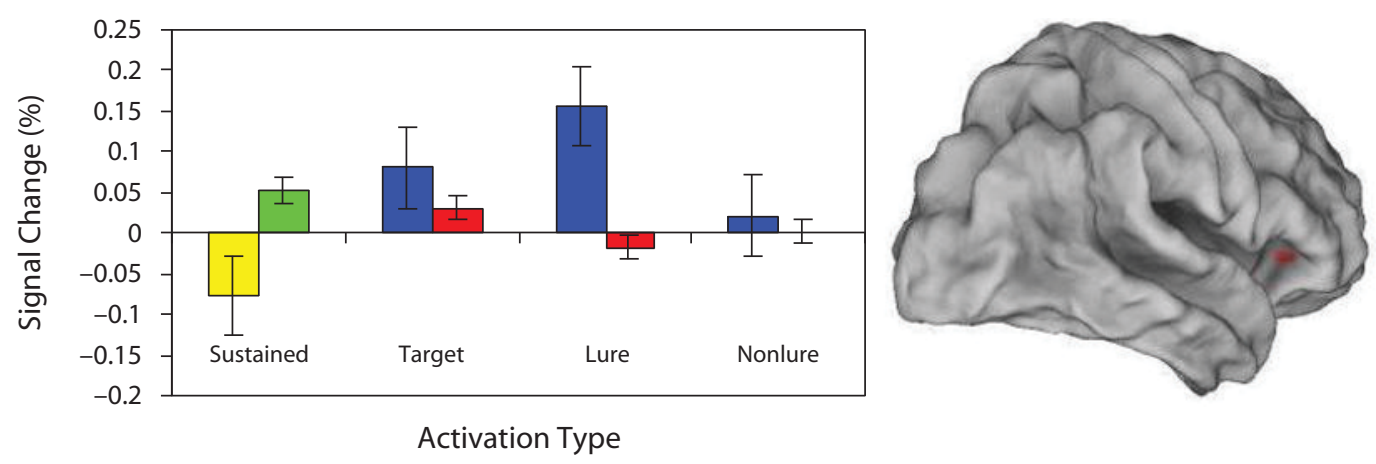

Left Inferior Parietal Lobe (BA 40)
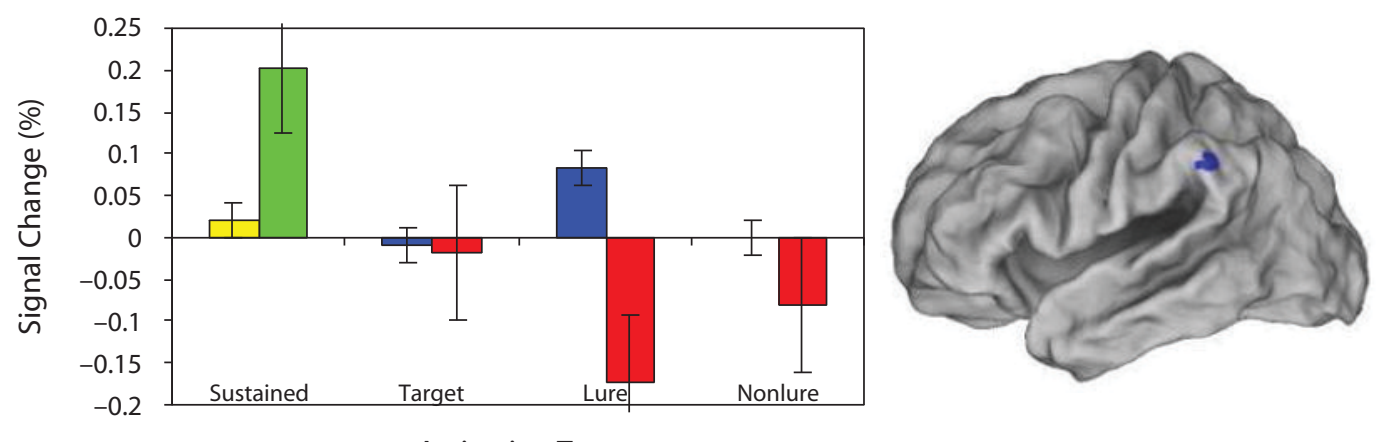

Activation Type

\begin{abstract}
Figure 5. Sustained and transient activation for two regions showing a significant trial type $\times$ anxiety group interaction in the neutral condition. The regions are (top) right ventrolateral PFC (peak Talairach coordinates: $33,28,-3)$ and (bottom) left inferior parietal lobe (peak Talairach coordinates: $-57,-47,35$ ). In the graphs, sustained activation is shown in yellow (for high-anxious groups) or green (for low-anxious groups). Transient trial-related activation is shown in blue (for high-anxious groups) or red (for low-anxious groups). Error bars show standard errors of the means.
\end{abstract}

type effect in this region, but the effect was in the opposite direction (activation rather than deactivation).

\section{Negative Mood Condition}

To examine the effects of the mood manipulation, we conducted a four-way ANOVA with video $\times$ network $X$ signal $\times$ group as factors. This analysis found that the four-way interaction achieved significance at the .05 level $\left[F(1,38)=3.97, p=.054, \eta^{2}=.09\right]$. As is shown in the bottom panel of Figure 3, in the negative condition (in contrast to the neutral condition), the two-way interactions of signal $\times$ group were no longer significant in either network $(p>9)$. Indeed, no group-related effects of any kind were significant in the negative condition. Examining each network separately across video conditions, we found that the three-way interaction of video $\times$ signal $\times$ group was significant for the cognitive control network $\left[F(1,38)=7.96, p=.008, \eta^{2}=.17\right]$, whereas in the default network there was a trend for a three-way interaction $\left[F(1,38)=2.92, p=.096, \eta^{2}=.07\right]$.

Post hoc paired-sample comparisons for each group separately revealed that only the low-anxious group showed significant activation changes as a result of the negative video: They significantly increased their transient activa- tion in the cognitive-control network $[t(19)=-2.98, p=$ $.008, \eta^{2}=.33$ ], but their decrease in sustained activation was not significant. Neither group showed significant changes in default-network recruitment. Finally, we also conducted correlation analyses $(n=96)$ to examine the fate of anxiety-related correlations as a result of the negative video. In the negative condition, anxiety scores were no longer significantly associated with sustained or transient response in either network ( $p \mathrm{~s}>.5)$.

Region-specific analyses were also conducted for the negative mood condition. In individual regions where group differences and correlational effects had been found in the neutral condition, these effects were absent after the negative video. (See the supplementary information for details.)

\section{Effects of Depression and Gender}

Because the high-anxiety group had significantly higher BDI scores than did the low-anxiety group, and also contained a disproportionate number of women, we looked for possible effects of these variables on the other results reported for the composite networks. To test our results for effects of depression, we conducted a two-factor ANCOVA on each network in the neutral condition, using 
signal type and group as factors, and BDI score as the covariate. For the cognitive-control network, the interaction of signal $\times$ group remained significant $[F(1,37)=$ $\left.7.86, p=.008, \eta^{2}=.18\right]$. For the default network, the interaction of signal $\times$ group now showed a trend toward significance $\left[F(1,37)=3.76, p=.06, \eta^{2}=.09\right]$. Thus, the effect of anxiety on the pattern of sustained and transient recruitment in both networks was relatively independent of depression scores.

We similarly looked for any effects of gender on this recruitment. Using gender as a covariate (Cohen \& Cohen, 1983), we found that in the cognitive-control network, the interaction of signal $\times$ group remained significant, $\left[F(1,37)=6.78, p=.013, \eta^{2}=.16\right]$. Likewise, in the default network, removing the effects of gender left the interaction of signal $\times$ group significant $[F(1,37)=6.54$, $\left.p=.015, \eta^{2}=.15\right]$. These results are consistent with analyses of POMS scores for the "anxious" and "gloomy" measures that were reported after the neutral and negative videos. We found no interaction between gender and video for these POMS scores, either alone or when entering anxiety scores as a covariate. Thus, these two confounding variables (depression, gender) were not the primary source of anxiety effects in these networks.

\section{DISCUSSION}

This study examined the neural basis of anxiety effects on cognitive efficiency. Using a mixed design to isolate sustained from transient activation, we found that, compared with low-anxious individuals, the high-anxious group showed significantly reduced sustained activity in cognitive control regions but increased transient activation during task trials. Similar results were found when correlations were conducted on continuous anxiety levels across the full sample. Importantly, these relationships were found in the absence of anxiety-related decreases in performance, and are consistent with the theory (Bishop, 2007) that higher anxiety reduces cognitive efficiency by impairing the ability to recruit working memory regions in what may be an optimal manner. In addition, the greater use of transient recruitment as anxiety increased is consistent with more traditional views of cognitive efficiency that emphasize brain regions "working harder" to perform at levels equivalent to those of nonanxious participants.

These results are largely consistent with the predictions of the DMC theory (Braver et al., 2007) in indicating that high-anxious individuals may reduce proactive control and increase reactive control during $n$-back task performance. In the $n$-back task, proactive control might be exerted by using a processing mode in which working memory mechanisms are recruited continuously across a block of trials. Such a mode would be advantageous in enabling ongoing maintenance of task stimuli across successive trials, which is required, given the continuous storage and updating demands on working memory present in the $n$-back task. Additionally, the DMC theory holds that a major function of proactive control is to help prevent or to manage interference during task processing using selective attention mechanisms. Although this inter- pretation is speculative, the set of regions found to show anxiety-group differences (see Table A2) fit well with this hypothesis. (For a discussion relating these regions to proactive interference management, see the supplementary information.)

The data we have presented also support the hypothesis that high-anxious individuals may increase reactive control in order to compensate for reductions in proactive control. Reactive control is thought to be critical for transiently detecting and resolving interference when it appears; thus, reactive control might be engaged preferentially for the high-interference lure trials. In our data, an analysis of activation $\times$ trial type indicated that in the right VLPFC (BA 47), the high-anxious individuals showed both a reduction in sustained activation as well as an increase in transient activation that occurred specifically on the high-interference lure trials. Right VLPFC has been linked generally to response inhibition and interference resolution (Aron, Robbins, \& Poldrack, 2004; Badre \& Wagner, 2005; Burgess \& Banich, 2008; Burgess $\&$ Braver, 2008) and more specifically to greater activation during retrieval of nontargets versus targets (Wolf, Vasic, \& Walter, 2006). The latter finding may be because nontargets include lure trials, or perhaps merely because right VLPFC is sensitive to multiple sources of information that must be evaluated, regardless of conflict (Badre $\&$ Wagner, 2005). Thus, a plausible interpretation for our trial-type effects is that low-anxious individuals tonically engaged right VLPFC in order to manage, or perhaps prevent, cognitive interference. As a consequence, there may have been no need to recruit this region further when individual lure trials occurred. By contrast, because of a reduced utilization of proactive control, the high-anxious group may have recruited right VLPFC more strongly in a transient manner when interference trials occurred. Because of the unequal proportion of lure versus nonlure trials, these findings should be interpreted with caution. Nevertheless, these data are consistent with the DMC theory that suggests anxious individuals should have a greater tendency to utilize cognitive control in a reactive rather than a proactive manner.

\section{Anxiety Effects on Default-Network Activation Levels}

A more surprising result was found in the anxietyrelated recruitment of default regions. Activity here, as in cognitive-control regions, showed significant group effects of trait anxiety on sustained activation. However, in the default regions, the effect was driven by the highanxiety participants showing greater sustained reductions in activation (deactivation) in default-network areas, whereas the low-anxious group showed only minimal sustained reductions in these areas. The extensive sustained reductions shown by the high-anxious group are contrary to what existing data on emotional modulation would predict. Most studies have found increased activation (loss of deactivation) as a result of negative trait emotion or emotional stimulation (Zald et al., 2002). In the present results, we see two possible interpretations: The reduced activation (stronger deactivation) in default regions may 
serve either a cognitive or an emotional function for the high-anxious group. As a cognitive mechanism, defaultmode deactivation may reflect an active inhibitory mechanism that serves to benefit performance. This notion is plausible because one function imputed to the default system is mediation of spontaneous mental activity such as visual imagery, sensory awareness, awareness of bodily state, or replaying of episodic memories (Christoff, Ream, \& Gabrieli, 2004; Mazoyer et al., 2001). The effect of default system deactivation during cognitive tasks would thus be to suspend this spontaneous activity and thereby facilitate cognitive processing. Reduced activation (stronger deactivation) has been shown to increase with task difficulty (McKiernan et al., 2003), and in ventromedial PFC specifically, it has been linked with performance improvements in the presence of anxious arousal (Simpson, Snyder, et al., 2001). It may be that trait-anxious individuals show greater sustained deactivation in these areas because they are applying greater cognitive effort in order to enhance performance.

Alternatively, the greater sustained reductions in default region activity could represent a mechanism that is fundamentally emotional in nature. We propose that defaultmode deactivation might reflect an implicit attempt to inhibit awareness of anxious arousal, or possibly to shut off explicit rumination. The medial PFC regions, especially ventrally, are thought to mediate emotional experiences and arousal (Drevets, 2001). Reducing activation in emotional regions may be a response that occurs when a cognitive task or other unfamiliar situation increases anxious arousal (particularly in a gradual or unattended manner). Simpson, Snyder, et al. (2001) proposed that ventromedial PFC could be modulated by both cognitive performance and emotional stimuli, and that this modulation might have a limited range. Thus, low-intensity emotional stimuli, or a cognitive task of moderate difficulty, could initially lead to deactivation of ventromedial PFC and other defaultsystem regions. However, increasing cognitive task difficulty or emotional stimulation would eventually result in loss of deactivation (increased activation). The sustained deactivation of our high-anxious group, if interpreted as an active inhibitory process, suggests the possibility that trait anxiety may induce suppression of default-mode processing simply as a general compensatory strategy for dealing with elevated arousal.

This model of ventromedial PFC as responding to both cognitive and emotional stimulation would also be consistent with the differential effects of the negative mood manipulation on sustained activity in the high- and lowanxiety groups. After the negative video, group differences in default mode activation were eliminated due to reductions in sustained default activation shown by the low-anxious group and increases in default activation (less deactivation) shown by the high-anxious group (who had already shown extensive sustained deactivation in the neutral condition). The effects of the negative video on cognitive control areas were even more substantial. After the negative video, sustained activity in cognitive control regions in the low-anxious group, was now accompanied by significant increases in the tran- sient recruitment of additional cognitive-control regions. Thus, following the negative video, the brain activation pattern in the low-anxious was more similar to that exhibited by the high-anxious group in the neutral condition. Given these results, it is tempting to assume that the negative video actually increased negative affect in the low-anxious group, but other interpretations are possible. For example, results for the positive-video data (reported elsewhere) yielded findings similar to those shown for the negative video, suggesting that the negative-video effects may actually have been due to enhanced emotional arousal. In any case, these results demonstrate that both trait anxiety and induced anxiety (or perhaps induced emotional arousal) can modulate recruitment of cognitive-control brain regions.

\section{Rethinking Cognitive Efficiency}

The results of the present study raise questions about the conventional definition of processing efficiency. If one focused only on transient response, our results would argue for lesser efficiency in the anxious group, consistent with the processing efficiency theory (Eysenck \& Calvo, 1992). The performance of the high-anxious group was no different than that of the low-anxious group, but their recruitment of cognitive control areas was significantly greater. However, when sustained and transient effects are considered together, we can reasonably ask whether the anxious group shows lower efficiency, or simply an alternate route to the same behavioral performance. In the present data at least, the high-anxious group's transient recruitment of cognitive control regions was accompanied by sustained deactivation of default regions. Such deactivation in default areas has been observed with increasing task difficulty and has been hypothesized to mediate performance improvements (McKiernan et al., 2003; Simpson, Drevets, et al., 2001). Perhaps there are two routes to improved performance: by increasing sustained activation in cognitive control regions, or by increasing sustained deactivation in default areas. If so, the sustained default deactivation seen in the high-anxious group could as plausibly improve performance as the sustained cognitive-control activation seen in the low-anxious group, perhaps by promoting, respectively, reduced susceptibility to bottom-up distractors or increased top-down goal-directed processing.

These results resonate with Eysenck et al.'s (2007) attentional control theory, in which those authors posited that anxiety affects cognition by altering the balance between top-down and bottom-up attentional systems. We speculate that the use of primarily sustained or primarily transient activation in a given network may be one way in which individuals achieve a particular balance between these two forms of cognitive control. According to this notion, higher anxiety may have biased our participants toward a form of cognitive control emphasizing reductions in default-network activation rather than increased cognitive-control-network activation, a finding consistent with Eysenck et al.'s attentional control theory. Morefocused tests are required to characterize the recruitment dynamics of cognitive efficiency more clearly. 


\section{Limitations and Future Issues}

A potential limitation to this study is that our highanxious participants were significantly more depressed than the low-anxious participants were. However, after the effects of depression were removed, the effects of anxiety on neural activation patterns remained largely significant. In addition, a grouping of participants with highest and lowest BDI scores showed substantial overlap with our anxiety groups, but nevertheless did not elicit the group differences in sustained-versus-transient patterns reported here. Thus, although there is a strong overlap between depression and anxiety, the presence of depression itself does not seem to lead to the altered neural recruitment reported here. A second potential limitation in this study was the substantial gender imbalance, raising the possibility that the anxiety effects are sex-specific. However, gender effects did not seem to interact with anxiety in producing our results, nor did they explain our results on their own. Nevertheless, until further studies have clarified the role of gender in anxiety, caution is needed in interpreting these findings.

To investigate the generality of our findings on the transient use of cognitive control, future studies could observe anxious individuals while they perform tasks that provide explicit behavioral measures of proactive versus reactive processing (Braver et al., 2007; Paxton, Barch, Racine, \& Braver, 2007; Speer, Jacoby, \& Braver, 2003). In addition, the sustained effects we report here were estimated on a block level, with removal of transient components of participants' BOLD responses on a trial-by-trial basis. A useful adjunct to the present study would be to observe anxious and nonanxious groups with working memory tasks that included an explicit delay period within each trial. In such trials, the time course of delay-related activation in cognitive control regions would provide additional evidence that anxious individuals show more transient recruitment of these areas, whereas nonanxious participants do not.

\section{Summary}

This study has examined the effects of trait and state anxiety on neural mechanisms of cognitive control and performance efficiency. Results showed that high and low anxious individuals make strikingly different use of cognitive and default-network circuitry during performance of a cognitive task. In addition, low-anxious participants can be induced by a negative-mood manipulation to shift their neural recruitment patterns to more closely resemble those of the high-anxiety participants. These results indicate that an analysis of sustained versus transient neural activity may be an important factor in understanding cognition-emotion interactions.

\section{AUTHOR NOTE}

The authors thank three anonymous reviewers for very helpful comments on this article. We also thank Elizabeth Chrastil, Adrianne Wilkerson, Timothy Heaven, and Naomi Yodkovik for help with data collection. This work was supported by grants from the National Institute of Mental Health (R01 MH66088 to J.R.G. and T.S.B., R01 MH-66078 to T.S.B., and R01 MH-66031 to D.M.B.). Correspondence concerning this article should be addressed to C. L. Fales, Department of Psychology, Wash- ington University, Campus Box 1125, St. Louis, MO 63130 (e-mail: cfales@artsci.wustl.edu).

\section{REFERENCES}

Aron, A. R., Robbins, T. W., \& Poldrack, R. A. (2004). Inhibition and the right inferior frontal cortex. Trends in Cognitive Sciences, 8, 170-177.

BADRE, D., \& WAGNer, A. D. (2005). Frontal lobe mechanisms that resolve proactive interference. Cerebral Cortex, 15, 2003-2012.

Beck, A. T., Ward, C. H., Mendelson, M., Mock, J., \& Erbaugh, J. (1961). An inventory for measuring depression. Archives of General Psychiatry, 4, 561-571.

BIsHOP, S. J. (2007). Neurocognitive mechanisms of anxiety: An integrative account. Trends in Cognitive Sciences, 11, 307-316.

Bishop, S. [J.], Duncan, J., Brett, M., \& Lawrence, A. D. (2004). Prefrontal cortical function and anxiety: Controlling attention to threat-related stimuli. Nature Neuroscience, 7, 184-188.

Borkovec, T. D., \& Roemer, L. (1995). Perceived functions of worry among generalized anxiety disorder subjects: Distraction from more emotionally distressing topics? Journal of Behavior Therapy \& Experimental Psychiatry, 26, 25-30.

Boynton, G. M., Engel, S. A., Glover, G. H., \& Heeger, D. J. (1996). Linear systems analysis of functional magnetic resonance imaging in human V1. Journal of Neuroscience, 16, 4207-4221.

Brahmbhatt, S. B., Haut, K., Csernansky, J. G., \& Barch, D. M. (2006). Neural correlates of verbal and nonverbal working memory deficits in individuals with schizophrenia and their high-risk siblings. Schizophrenia Research, 87, 191-204.

Braver, T. S., Cohen, J. D., Nystrom, L. E., Jonides, J., Smith, E. E., \& Noll, D. C. (1997). A parametric study of prefrontal cortex involvement in human working memory. NeuroImage, 5, 49-62.

Braver, T. S., Gray, J. R., \& Burgess, G. C. (2007). Explaining the many varieties of working memory variation: Dual mechanisms of cognitive control. In A. R. A. Conway, C. Jarrold, M. J. Kane, A. Miyake, \& J. N. Towse (Eds.), Variation in working memory (pp. 76-106). Oxford: Oxford University Press.

Brown, T. A., Antony, M. M., \& Barlow, D. H. (1992). Psychometric properties of the Penn State worry questionnaire in a clinical anxiety disorders sample. Behaviour Research \& Therapy, 30, 33-37.

Buckner, R. L., Snyder, A. Z., Shannon, B. J., LaRossa, G., SACHs, R., Fotenos, A. F., ET AL. (2005). Molecular, structural, and functional characterization of Alzheimer's disease: Evidence for a relationship between default activity, amyloid, and memory. Journal of Neuroscience, 25, 7709-7717.

Burgess, G. C., \& BANICH, M. T. (2008). Investigation of sustained and transient interference control in the color-word Stroop task. Manuscript in preparation.

BurgESS, G. C., \& BRAVER, T. S. (2008). Relationship between general fluid intelligence and interference control: Evidence for dual mechanisms of cognitive control over interference in working memory. Manuscript in preparation.

Calvo, M. G. (1985). Effort, aversive representations and performance in test anxiety. Personality \& Individual Differences, 6, 563-571.

Calvo, M. G., \& Ramos, P. M. (1989). Effects of test anxiety on motor learning: The processing efficiency hypothesis. Anxiety Research, 2, 45-55.

Calvo, M. G., Ramos, P. M., \& Estevez, A. (1992). Test anxiety and comprehension efficiency: The role of prior knowledge and working memory deficits. Anxiety, Stress, \& Coping, 5, 125-138.

Cazalis, F., Valabrègue, R., Pélégrini-Issac, M., Asloun, S., Robbins, T. W., \& GRANON, S. (2003). Individual differences in prefrontal cortical activation on the Tower of London planning task: Implication for effortful processing. European Journal of Neuroscience, 17, 2219-2225.

Christoff, K., Ream, J. M., \& Gabrieli, J. D. E. (2004). Neural basis of spontaneous thought processes. Cortex, 40, 623-630.

CoHen, J., \& CoHen, P. (1983). Applied multiple regression/correlation analysis for the behavioral sciences. Hillsdale, NJ: Erlbaum.

Cohen, J., MacWhinney, B., Flatt, M., \& Provost, J. (1993). PsyScope: An interactive graphic system for designing and controlling experiments in the psychology laboratory using Macintosh computers. Behavior Research Methods, Instruments, \& Computers, 25, 257-271.

DARKE, S. (1988). Effects of anxiety on inferential reasoning task performance. Journal of Personality \& Social Psychology, 55, 499-505. 
DeraKshan, N., \& EysencK, M. W. (1998). Working memory capacity in high trait-anxious and repressor groups. Cognition \& Emotion, 12, 697-713.

Dolcos, F., \& McCarthy, G. (2006). Brain systems mediating cognitive interference by emotional distraction. Journal of Neuroscience, 26, 2072-2079.

Donaldson, D. I., Petersen, S. E., Ollinger, J. M., \& Buckner, R. L. (2001). Dissociating state and item components of recognition memory using fMRI. NeuroImage, 13, 129-142.

Donohue, S. E., Wendelken, C., \& Bunge, S. A. (2008). Neural correlates of preparation for action selection as a function of specific task demands. Journal of Cognitive Neuroscience, 20, 694-706.

Drevets, W. C. (2001). Neuroimaging and neuropathological studies of depression: Implications for the cognitive-emotional features of mood disorders. Current Opinion in Neurobiology, 11, 240-249.

Drevets, W. C., \& Raichle, M. E. (1998). Reciprocal suppression of regional cerebral blood flow during emotional versus higher cognitive processes: Implication for interactions between emotion and cognition. Cognition \& Emotion, 12, 353-385.

Eisenberger, N. I., Lieberman, M. D., \& Satpute, A. B. (2005). Personality from a controlled processing perspective: An fMRI study of neuroticism, extraversion, and self-consciousness. Cognitive, Affective, \& Behavioral Neuroscience, 5, 169-181.

Eysenck, M. W. (1982). Attention and arousal: Cognition and performance. Berlin: Springer.

Eysenck, M. W. (1985). Anxiety and cognitive-task performance. Personality \& Individual Differences, 6, 579-586.

Eysenck, M. W., \& Calvo, M. G. (1992). Anxiety and performance: The processing efficiency theory. Cognition \& Emotion, 6, 409-434.

Eysenck, M. W., Derakshan, N., Santos, R., \& Calvo, M. G. (2007). Anxiety and cognitive performance: Attentional control theory. Emotion, 7, 336-353.

Fischer, H., Sandblom, J., Herlitz, A., Fransson, P., Wright, C. I., \& BäCKMAN, L. (2004). Sex-differential brain activation during exposure to female and male faces. NeuroReport, 15, 235-238.

Fox, E., \& Georgiou, G. A. (2005). The nature of attentional bias in human anxiety. In R. W. Engle, G. Sedek, U. von Hecker, \& D. N. McIntosh (Eds.), Cognitive limitations in aging and psychopathology (pp. 249-274). Cambridge: Cambridge University Press.

Friston, K. J., Williams, S. R., Howard, R., Frackowiak, R. S. J., \& TURNER, R. (1996). Movement-related effects in fMRI time-series. Magnetic Resonance in Medicine, 35, 346-355.

Gray, J. R. (2001). Emotional modulation of cognitive control: Approach-withdrawal states double-dissociate spatial from verbal two-back task performance. Journal of Experimental Psychology: General, 130, 436-452.

Gray, J. R., Braver, T. S., \& Raichle, M. E. (2002). Integration of emotion and cognition in the lateral prefrontal cortex. Proceedings of the National Academy of Sciences, 99, 4115-4120.

Gray, J. R., Burgess, G. C., Schaefer, A., Yarkoni, T., Larsen, R. J., \& BraVER, T. S. (2005). Affective personality differences in neural processing efficiency confirmed using fMRI. Cognitive, Affective, \& Behavioral Neuroscience, 5, 182-190.

Gray, J. R., Chabris, C. F., \& Braver, T. S. (2003). Neural mechanisms of general fluid intelligence. Nature Neuroscience, 6, 316-322.

Greicius, M. D., Krasnow, B., Reiss, A. L., \& Menon, V. (2003). Functional connectivity in the resting brain: A network analysis of the default mode hypothesis. Proceedings of the National Academy of Sciences, 100, 253-258.

Gusnard, D. A., \& Raichle, M. E. (2001). Searching for a baseline: Functional imaging and the resting human brain. Nature Reviews Neuroscience, 2, 685-694.

Hamilton, V. (1978, December). The cognitive analysis of personality related to information-processing deficits with stress and anxiety. Paper presented at the British Psychological Society meeting, London.

Koster, E. H. W., Crombez, G., Verschuere, B., Van Damme, S., \& Wiersema, J. R. (2006). Components of attentional bias to threat in high trait anxiety: Facilitated engagement, impaired disengagement, and attentional avoidance. Behaviour Research \& Therapy, 44, 1757-1771.

Lancaster, J. L., Glass, T. G., Lankipalli, B. R., Downs, H., MaYBERG, H., \& Fox, P. T. (1995). A modality-independent approach to spatial normalization of tomographic images of the human brain. Human Brain Mapping, 3, 209-223.
LeON, M. R., \& Revelle, W. (1985). Effects of anxiety on analogical reasoning: A test of three theoretical models. Journal of Personality \& Social Psychology, 49, 1302-1315.

MADDOCK, R. J. (1999). The retrosplenial cortex and emotion: New insights from functional neuroimaging of the human brain. Trends in Neurosciences, 22, 310-316.

Maddock, R. J., Garrett, A. S., \& Buonocore, M. H. (2003). Posterior cingulate cortex activation by emotional words: fMRI evidence from a valence decision task. Human Brain Mapping, 18, 30-41.

Mazoyer, B., Zago, L., Mellet, E., Bricogne, S., Etard, O., HoudÉ, O., ET AL. (2001). Cortical networks for working memory and executive functions sustain the conscious resting state in man. Brain Research Bulletin, 54, 287-298.

McKiernan, K. A., Kaufman, J. N., Kucera-Thompson, J., \& Binder, J. R. (2003). A parametric manipulation of factors affecting taskinduced deactivation in functional neuroimaging. Journal of Cognitive Neuroscience, 15, 394-408.

Meyer, T. J., Miller, M. L., Metzger, R. L., \& Borkovec, T. D. (1990). Development and validation of the Penn State Worry Questionnaire. Behaviour Research \& Therapy, 28, 487-495.

Owen, A. M., McMillan, K. M., Laird, A. R., \& Bullmore, E. [T.] (2005). N-back working memory paradigm: A meta-analysis of normative functional neuroimaging studies. Human Brain Mapping, 25, 46-59.

Paxton, J. L., Barch, D. M., Racine, C. A., \& Braver, T. S. (2007). Cognitive control, goal maintenance, and prefrontal function in healthy aging. Cerebral Cortex, 18, 1010-1028.

Richards, A., French, C. C., Keogh, E., \& Carter, C. (2000). Testanxiety, inferential reasoning and working memory load. Anxiety, Stress, \& Coping, 13, 87-109.

Schwartz, C. E., Wright, C. I., Shin, L. M., Kagan, J., Whalen, P. J., McMullin, K. G., \& Rauch, S. L. (2003). Differential amygdalar response to novel versus newly familiar neutral faces: A functional MRI probe developed for studying inhibited temperament. Biological Psychiatry, 53, 854-862.

Shulman, G. L., Fiez, J. A., Corbetta, M., Buckner, R. L., Miezin, F. M., Raichle, M. E., \& Petersen, S. E. (1997). Common blood flow changes across visual tasks: II. Decreases in cerebral cortex. Journal of Cognitive Neuroscience, 9, 648-663.

Simpson, J. R., Jr., Drevets, W. C., Snyder, A. Z., Gusnard, D. A., \& RAICHLE, M. E. (2001). Emotion-induced changes in human medial prefrontal cortex: II. During anticipatory anxiety. Proceedings of the National Academy of Sciences, 98, 688-693.

Simpson, J. R., Jr., Snyder, A. Z., Gusnard, D. A., \& Raichle, M. E. (2001). Emotion-induced changes in human medial prefrontal cortex: I. During cognitive task performance. Proceedings of the National Academy of Sciences, 98, 683-687.

SNYDER, A. Z. (1996). Difference image versus ratio image error function forms in PET-PET realignment. In R. Myers, V. Cunningham, D. Bailey, \& T. Jones (Eds.), Quantification of brain function using PET (pp. 131-137). San Diego: Academic Press.

SPeER, N. K., Jacoby, L. L., \& Braver, T. S. (2003). Strategy-dependent changes in memory: Effects on behavior and brain activity. Cognitive, Affective, \& Behavioral Neuroscience, 3, 155-167.

TAlairach, J., \& Tournoux, P. (1988). Co-planar stereotaxic atlas of the human brain: 3-Dimensional proportional system. An approach to cerebral imaging (M. Rayport, Trans.). Stuttgart: Thieme.

TAYLOR, J. A. (1953). A personality scale of manifest anxiety. Journal of Abnormal \& Social Psychology, 48, 285-290.

TOHILl, J. M., \& HolyoAK, K. J. (2000). The impact of anxiety on analogical reasoning. Thinking \& Reasoning, 6, 27-40.

Visscher, K. M., Miezin, F. M., Kelly, J. E., Buckner, R. L., DonALdson, D. I., McAvoy, M. P., ET AL. (2003). Mixed blocked/eventrelated designs separate transient and sustained activity in fMRI. NeuroImage, 19, 1694-1708.

WAGER, T. D., \& SMITH, E. E. (2003). Neuroimaging studies of working memory: A meta-analysis. Cognitive, Affective, \& Behavioral Neuroscience, 3, 255-274.

Wagner, A. D., Maril, A., Bjork, R. A., \& Schacter, D. L. (2001). Prefrontal contributions to executive control: fMRI evidence for functional distinctions within lateral prefrontal cortex. Neurolmage, 14, 1337-1347.

Wedig, M. M., Rauch, S. L., Albert, M. S., \& Wright, C. I. (2005). 
Differential amygdala habituation to neutral faces in young and elderly adults. Neuroscience Letters, 385, 114-119.

Weissman, D. H., Roberts, K. C., Visscher, K. M., \& WoldorfF, M. G. (2006). The neural bases of momentary lapses in attention. $\mathrm{Na}$ ture Neuroscience, 9, 971-978.

Wolf, R. C., Vasic, N., \& Walter, H. (2006). Differential activation of ventrolateral prefrontal cortex during working memory retrieval. Neuropsychologia, 44, 2558-2563.

Wright, P., \& LIU, Y. (2006). Neutral faces activate the amygdala during identity matching. NeuroImage, 29, 628-636.

Zald, D. H., Mattson, D. L., \& PARdo, J. V. (2002). Brain activity in ventromedial prefrontal cortex correlates with individual differences in negative affect. Proceedings of the National Academy of Sciences, 99, 2450-2454.

Zarantonello, M., Slaymaker, F., Johnson, J. E., \& Petzel, T.
(1984). Effects of anxiety and depression on anagram performance, ratings of cognitive interference, and the negative subjective evaluation of performance. Journal of Clinical Psychology, 40, 20-25.

\section{NOTES}

1. To minimize affect-related confounds due to stimulus type, we excluded face data from analyses because faces are not always affectively neutral. Viewing faces, even neutral ones, often evokes automatic amygdala responses (Fischer et al., 2004; Schwartz et al., 2003; Wedig, Rauch, Albert, \& Wright, 2005; Wright \& Liu, 2006).

2. At the time of the Shulman et al. (1997) study, it was standard practice to express $\mathrm{rCBF}$ change relative to the whole brain mean rather than to the local baseline signal.

\section{APPENDIX}

Table A1

Centers of Mass for Regions of Interest (ROIs)

Used to Mask the Neuroimaging Data

\begin{tabular}{|c|c|c|c|c|}
\hline Study & $x$ & $y$ & $z$ & $\mathrm{~mm}^{3}$ \\
\hline \multicolumn{5}{|l|}{ Owen et al. (2005) } \\
\hline \multirow[t]{2}{*}{ Lateral premotor (6) } & 28 & 0 & 52 & 20-mm sphere \\
\hline & -26 & 2 & 52 & 20-mm sphere \\
\hline Dorsal cingulate/SMA $(32,6)$ & -2 & 12 & 42 & 20-mm sphere \\
\hline $\operatorname{DLPFC}(46,9)$ & 42 & 32 & 30 & 20-mm sphere \\
\hline \multirow[t]{2}{*}{ Ventrolateral PFC (44) } & -50 & 12 & 8 & 20-mm sphere \\
\hline & -62 & 0 & 14 & 20-mm sphere \\
\hline \multirow[t]{2}{*}{ Frontal pole (10) } & -38 & 44 & 20 & 20-mm sphere \\
\hline & 36 & 46 & 18 & 20-mm sphere \\
\hline Medial posterior parietal (7) & 12 & -64 & 48 & 20-mm sphere \\
\hline \multirow[t]{3}{*}{ Inferior parietal lobe (40) } & 30 & -58 & 42 & 20-mm sphere \\
\hline & 38 & -46 & 38 & 20-mm sphere \\
\hline & -34 & -48 & 38 & 20-mm sphere \\
\hline \multicolumn{5}{|l|}{ Wager \& Smith (2003) } \\
\hline BA $10,9,46,47$ & -32 & 34 & 22 & 20-mm sphere \\
\hline BA 9,6 & -45 & 7 & 32 & 20-mm sphere \\
\hline BA $40,39,7$ & -37 & -51 & 41 & 20-mm sphere \\
\hline BA $9,10,46$ & 36 & 36 & 28 & 20-mm sphere \\
\hline BA 7,40 & 31 & -59 & 43 & 20-mm sphere \\
\hline $\mathrm{BA} 47,10,11,13$ & 34 & 31 & -4 & 20-mm sphere \\
\hline BA 7 & -12 & -70 & 46 & 20-mm sphere \\
\hline BA $6,32,8$ & 0 & 11 & 49 & 20-mm sphere \\
\hline BA 6 & 27 & 0 & 56 & 20-mm sphere \\
\hline BA $6,9,44$ & 45 & 1 & 29 & 20-mm sphere \\
\hline BA 6 & -28 & -4 & 56 & 20-mm sphere \\
\hline \multicolumn{5}{|l|}{ Shulman et al. (1997) } \\
\hline Posterior cing./precuneus $(31,7)$ & -7 & -53 & 34 & \\
\hline Left inferior parietal ctx (40) & -57 & -35 & 40 & \\
\hline Left inferior parietal ctx $(39,19)$ & -43 & -69 & 36 & \\
\hline Right inferior parietal ctx (40) & 49 & -55 & 40 & \\
\hline Left lateral PFC (8) & -29 & 29 & 40 & \\
\hline Left medial PFC $(8,9)$ & -9 & 43 & 42 & \\
\hline Right medial PFC $(8,9)$ & 11 & 43 & 44 & \\
\hline Left frontal pole (9) & -17 & 63 & 18 & \\
\hline Left frontal pole (10) & -19 & 57 & 8 & \\
\hline Medial PFC (10) & -1 & 49 & -6 & \\
\hline Left ventral lateral PFC $(10,47)$ & -35 & 47 & -6 & \\
\hline Ventral medial PFC (32) & -3 & 19 & -14 & \\
\hline Left inferior temporal lobe (20) & -47 & -23 & -16 & \\
\hline Right amygdala & 17 & -7 & -18 & \\
\hline
\end{tabular}

Note-See the Method section for details. 
APPENDIX (Continued)

Table A2

Regions Showing Significant Group Differences in the Neutral Condition

\begin{tabular}{|c|c|c|c|c|c|c|c|c|c|c|}
\hline Region (BA) & Voxels & Response & Type & $x$ & $y$ & $z$ & Group Effect & $z$-score & Interaction & $r$ \\
\hline R-VLPFC & 14 & Sustained & $\mathrm{CC}$ & 26 & 28 & -3 & Low $>$ High & 2.90 & 2.35 & $-.288^{* *}$ \\
\hline L-IFG (44/45) & 16 & Sustained & $\mathrm{CC}$ & -59 & 5 & 7 & Low $>$ High & 2.56 & NS & $-.239^{*}$ \\
\hline R-MFG (46/10) & 26 & Sustained & $\mathrm{CC}$ & 42 & 47 & 15 & Low $>$ High & 2.47 & NS & $-.276^{* *}$ \\
\hline $\mathrm{mPFC} / \mathrm{D}-\mathrm{ACC}(6 / 32)$ & 232 & Sustained & $\mathrm{CC}$ & 0 & 12 & 45 & Low $>$ High & 2.85 & NS & $-.356^{* *}$ \\
\hline R-IPL 40 (SMG) & 47 & Sustained & $\mathrm{CC}$ & 32 & -64 & 39 & Low > High & 2.58 & NS & $-.285^{* *}$ \\
\hline L-Precun & 110 & Sustained & $\mathrm{CC}$ & -10 & -69 & 44 & Low > High & 2.97 & NS & $-.310^{* *}$ \\
\hline R-Precun & 96 & Sustained & $\mathrm{CC}$ & 11 & -63 & 48 & Low $>$ High & 2.90 & NS & $-.279^{* *}$ \\
\hline L-Parahipp.G/Amyg & 25 & Sustained & Def. & -29 & 0 & -16 & Low $>$ High & 2.61 & NS & -.135 \\
\hline $\mathrm{L}-47 / 10$ & 11 & Sustained & Def. & -26 & 44 & -7 & Low $>$ High & 2.70 & NS & $-.269^{* *}$ \\
\hline Pregen ACC 32 & 31 & Sustained & Def. & -1 & 43 & -2 & Low $>$ High & 2.47 & NS & $-.255^{* *}$ \\
\hline R-IPL 40/39 & 310 & Sustained & Def. & 50 & -56 & 25 & Low $>$ High & 3.56 & NS & $-.374^{* *}$ \\
\hline L-Precun 7/31 & 221 & Sustained & Def. & -3 & -48 & 32 & Low $>$ High & 2.81 & NS & $-.343^{* *}$ \\
\hline L-IPL 40/39 & 293 & Sustained & Def. & -47 & -61 & 27 & Low > High & 3.54 & NS & $-.383^{* *}$ \\
\hline mPFC 9 & 110 & Sustained & Def. & -1 & 43 & 33 & Low $>$ High & 2.52 & NS & $-.246^{* *}$ \\
\hline L-SFG/MFG 8 & 70 & Sustained & Def. & -25 & 22 & 46 & Low > High & 2.59 & NS & $-.225^{*}$ \\
\hline R-SFG 8 & 16 & Sustained & Def. & 19 & 23 & 49 & Low $>$ High & 2.34 & NS & $-.240^{*}$ \\
\hline IFG/Prec. 6/44 & 94 & Transient & $\mathrm{CC}$ & -54 & 7 & 7 & High $>$ Low & 3.46 & 3.06 & $+.395^{* *}$ \\
\hline MFG 46 & 58 & Transient & $\mathrm{CC}$ & -33 & 40 & 16 & High $>$ Low & 3.03 & 2.69 & $+.374^{* *}$ \\
\hline MFG 46 & 139 & Transient & $\mathrm{CC}$ & 38 & 34 & 28 & High $>$ Low & 3.41 & 2.80 & $+.401^{* *}$ \\
\hline IFG 44 & 13 & Transient & $\mathrm{CC}$ & 41 & 6 & 32 & High > Low & 2.75 & NS & $+.351^{* *}$ \\
\hline IPL 40 & 95 & Transient & $\mathrm{CC}$ & -32 & -48 & 42 & High $>$ Low & 3.17 & 3.15 & $+.350^{* *}$ \\
\hline D-ACC 32 & 100 & Transient & $\mathrm{CC}$ & 2 & 9 & 44 & High $>$ Low & 3.03 & 3.52 & $+.358^{* *}$ \\
\hline MFG 9/Prec. 6 & 27 & Transient & $\mathrm{CC}$ & -44 & 6 & 37 & High $>$ Low & 3.02 & NS & $+.294^{* *}$ \\
\hline PCU 7 & 10 & Transient & $\mathrm{CC}$ & -5 & -66 & 39 & High > Low & 2.96 & NS & $+.380^{* *}$ \\
\hline PCU 7 & 19 & Transient & $\mathrm{CC}$ & 15 & -64 & 40 & High > Low & 3.01 & 2.78 & $+.446^{* *}$ \\
\hline MFG 6/8/D-ACC 32 & 25 & Transient & $\mathrm{CC}$ & 25 & 5 & 46 & High > Low & 2.83 & 3.29 & $+.357^{* *}$ \\
\hline MFG 6/mPFC 6 & 81 & Transient & $\mathrm{CC}$ & -22 & 0 & 48 & High $>$ Low & 3.34 & 2.85 & $+.392^{* *}$ \\
\hline L-MFG 10/ACC 32 & 16 & Transient & Def. & -23 & 47 & -2 & High $>$ Low & 3.13 & NS & $+.314^{* *}$ \\
\hline L-IPL 40 & 16 & Transient & Def. & -55 & -38 & 27 & High > Low & 3.09 & NS & $+.269^{* *}$ \\
\hline R-IPL 40 & 18 & Transient & Def. & 57 & -30 & 32 & High > Low & 3.45 & NS & $+.272^{* *}$ \\
\hline R-PCU 7 & 11 & Transient & Def. & 5 & -48 & 33 & High > Low & 2.78 & NS & $+.377^{* *}$ \\
\hline Post. cingulate 31 & 8 & Transient & Def. & 0 & -34 & 37 & High > Low & 2.76 & NS & $+.339^{* *}$ \\
\hline
\end{tabular}

Note-Regions showing significant group differences in the neutral condition. For the sustained response, the high-anxious group shows less activity in all regions than the low-anxious group. In the cognitive control regions, this is because the low-anxious participants are more active than the high-anxious participants. In the default regions, this difference is because the high-anxious participants are more deactivated than the low-anxious participants. For the transient response, the high-anxious group shows greater activation in all regions than the low-anxious group. In the cognitive control regions, this is because the high-anxious participants are more active than the low-anxious participants. In the default regions, this is because the high-anxious participants are more active (not deactivated at all) than the low-anxious participants. "CC" denotes a cognitive control region. "Def." denotes a default-system region. Group differences for these regions were significant in the neutral condition, but not in the anxiety condition (as compared by independent $t$ tests). The "Interaction" column gives $z$-scores for regions where the interaction of video type $\times$ group in a two-way ANOVA is significant at .01 (for sustained response) or .005 (for transient response). The $r$ column gives correlation coefficients between anxiety scores and neural activity across the full sample of 96 participants. ${ }^{*} p<.05 . \quad{ }^{* *} p<.01$.

(Manuscript received June 29, 2007;

revision accepted for publication April 2, 2008.) 